\title{
Preliminary results from the EUSO-Balloon flight
}

\section{The JEM-EUSO Collaboration*}

E-mail: bertaina@to.infn.it

EUSO-Balloon was launched by the French Space Agency CNES from the Timmins base in Ontario (Canada) on the moonless night of August 25, 2014 UT. After reaching the floating altitude of about $38 \mathrm{~km}$, EUSO-Balloon imaged the UV background in the wavelength range 290 - $430 \mathrm{~nm}$ for more than 5 hours before descending to ground using the key technologies of JEM-EUSO. A detailed and precise measurement of the UV background in different atmospheric and ground conditions was achieved. The instrument proved the capability of detecting Extensive Air Showers by observing laser tracks with similar characteristics. The on going analyses of EUSO-Balloon data and their preliminary results are presented.

The 34th International Cosmic Ray Conference,

30 July- 6 August, 2015

The Hague, The Netherlands

${ }^{*}$ Speaker. 


\section{Introduction}

The EUSO-Balloon experiment [1] is a pathfinder mission for JEM-EUSO (Extreme Universe Space Observatory on-board the Japanese Experimental Module) [2]. The main objectives of the EUSO-Balloon pathfinder are to perform: a) a full scale end-to-end test of all the key technologies and instrumentation of JEM-EUSO detectors; b) a detailed and precise measurement of the UV background in different atmospheric and ground conditions; c) a first measurement of air shower tracks from the edge of space. For its first flight, EUSO-Balloon was launched by the French Space Agency CNES from the Timmins base in Ontario (Canada) on the moonless night of August 25, 2014 UT [3]. After reaching the floating altitude of about $38 \mathrm{~km}$, EUSO-Balloon imaged the UV background in the wavelength range $290-430 \mathrm{~nm}$ for more than 5 hours before descending to ground. The refractor telescope consisted of two Fresnel lenses and a focal surface filled with multi-anode photomultipliers. The spatial and temporal resolutions of the detector were $\sim 130 \mathrm{~m}$ and $2.5 \mu \mathrm{s}$, respectively. The full field of view in nadir mode was about 11 degrees. The UV data were complemented by Infrared images taken by an infrared camera on board EUSO-Balloon [4]. During 2.5 hours of EUSO-Balloon flight, a helicopter circled under the balloon operating UV flashers [5] and a UV laser [6] to simulate the optical signals from extreme energy cosmic rays, to calibrate the apparatus, and to characterise the optical atmospheric conditions. During flight EUSO-Balloon took more than 2.5 million images that are currently under analysis to infer different information: study of the performance of the different parts of the detector; response of the detector to the UV flasher and laser events; UV radiance from the Earth atmosphere and ground in different conditions: clear and cloudy atmosphere, grass, forests, lakes, as well as city lights. In parallel the data of the IR camera are used to localize clouds and estimate their cloud-top.

\section{The Flight}

The flight trajectory of EUSO-Balloon is shwon in Fig. 1. During more than five hours of operation at float, EUSO-Balloon recorded and stored on the two redundant hard-drives on board a total of 258,592 data-packets, corresponding to roughly 33 million frames (GTUs). A variety of ground covers have been over flown, including different types of soil and vegetation, wetlands, open water, urban and industrial areas to characterise the background intensity in several conditions. During its flight EUSO-Balloon crossed areas characterised by scattered and broken clouds at low altitudes (around $700-800 \mathrm{hPa}$ ) and ice thick clouds at higher altitudes (around $200-300 \mathrm{hPa}$ ). A detailed analysis of the atmospheric conditions is reported in [7, 8]. All this variety of situations turned out to be an ideal case to test the conditions that JEM-EUSO is expected to view during its orbits on the ISS. Moreover, to calibrate and reproduce artificially air shower-tracks a system consisting of a pulsed UV laser and two UV flashers (LED and Xe) flew on a Bell 212 helicopter below EUSO-Balloon for more than 2 hours between 03:31 and 05:52 UT. The wavelengths of these sources were chosen to mirror the fluorescence emission of electrons in air. The nominal laser energy was equivalent to the light emitted by a $100 \mathrm{EeV}$ EAS [9]. During this time the sources were fired $\sim 150,000$ times with two energy settings (see [5, 6] for details). The laser energy was changed every two minutes between $15 \mathrm{~mJ}$ and $10 \mathrm{~mJ}$ and was fired at a rate of $19 \mathrm{~Hz}$. This repetition rate was chosen to guarantee random coincidences between the readout of the balloon 


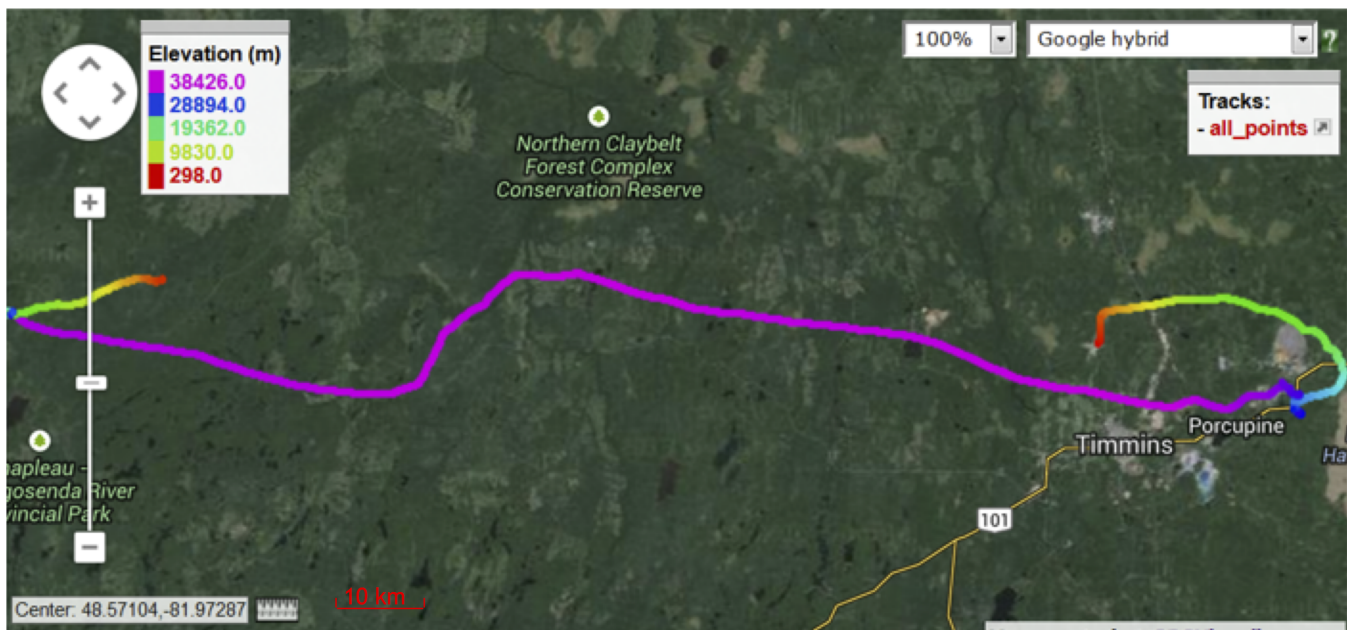

Figure 1: The flight-trajectory of EUSO-Balloon on August 25, 2014 UT. The flight started from the Timmins airport and ended in a tiny lake on the left part of the figure. The elevation of EUSO-Balloon during flight is indicated in the legend. In the first part of the flight EUSO-Balloon imaged mostly urban areas (Porcupine, Timmins, etc.) while the second part of the flight was mostly on forests and lakes.

$(20 \mathrm{~Hz})$ and the laser shots. This was necessary because there was no time synchronisation between the two systems. Around 300 tracks were found in EUSO-Balloon data. An example of such a light sequence is displayed in Fig. 2. The integrated number of counts in a typical packet ( $R U N=043202$, packet=1960) in which all the light sequence was imaged is shown in the left plot. The led and Xeflasher signals are located around pixel with coordinates $(\mathrm{X}=5 ; \mathrm{Y}=25)$. The evolution of the signal in the $3 \times 3$ pixel-box centred around $(X=5 ; Y=25)$ during the entire packet is displayed in the right plot. The led light appears between GTUs 19 - 31, followed by the laser shot at GTUs 55 - 56 and by the Xe-flasher between GTUs 58 - 65. An after pulse from the Xe-flasher occurs between GTUs $70-73$. The light sequence was synchronised in the following way. First, a UV led signal was shot for 12 GTUs with increasing luminosity to achieve a projected number of photoelectrons at PDM level raising from $\sim 1$ to $\sim 50$ counts. The sequence of led intensities was kept constant during the entire flight. This light-signal appears on the FS as a static source and can be used to determine the position of the helicopter in the FoV. About 25 GTUs after the end of the led signal, a $\sim 5 \mathrm{~mJ}$ laser shot lasting $7 \mathrm{~ns}$ was fired. The laser event took at maximum 10 GTUs to cross the entire FoV of the telescope. A Xe-flasher lamp was finally fired $\sim 5 \mu$ s after the laser shot for a 8 GTU duration. The variable light intensity of the Xe-flashers was reaching a maximum after the first 3 GTUs and then decreasing for the remaining time. Four different absolute intensities were used to mimic different energies of vertical showers.

\section{Technological Aspects}

The main objective of the first flight was to operate a full scale end-to-end test of all the key technologies and instrumentation of JEM-EUSO detectors. A detailed description of the instrument is reported in [3]. Different configurations of the photomultipliers' gain and thresholds on the photon-counting were applied. The acquisition system performed rather well with an integrated 

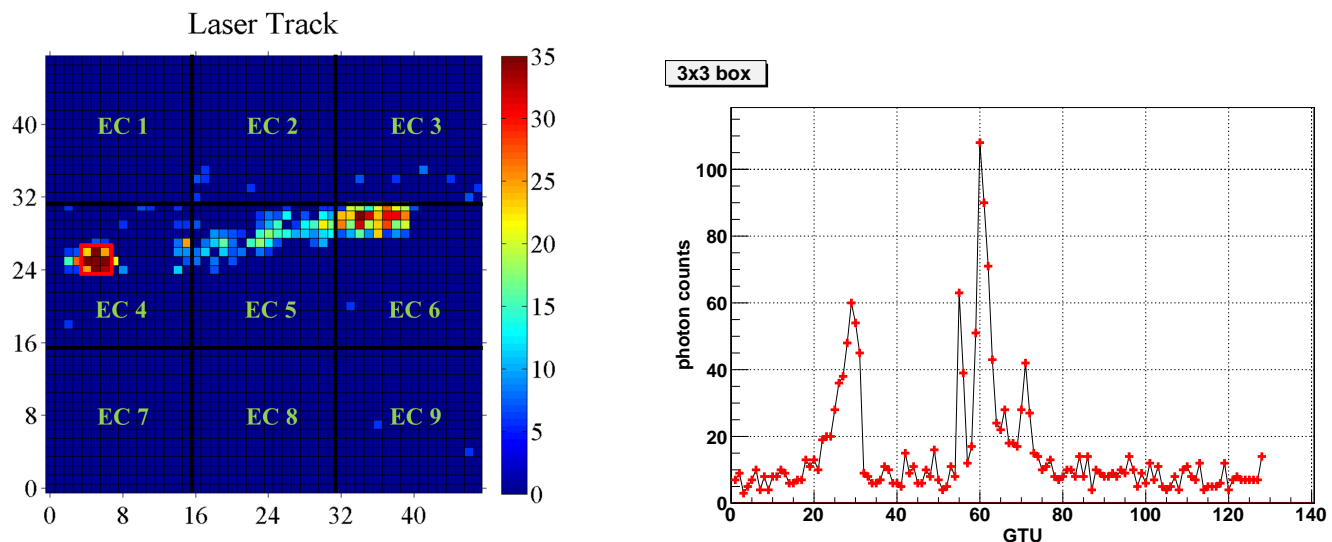

Figure 2: Left side: Image of one helicopter event obtained by integrating the counts in each pixel for the whole packet=1960 or RUN=043202 (128 GTUs). A threshold is applied to the minimum signal level to emphasize the location of the track. The UV-led and Xe-flasher signals are centred around pixel (axis of abscissae $X=5$; axis of ordinates $Y=25$ ). Right side: Number of counts recorded in the $3 \times 3$ pixel-box centered around $(\mathrm{X}=5 ; \mathrm{Y}=25)$ during the entire packet [21].

data taking of $15,300 \mathrm{~s}$ of the total time at float $18,900 \mathrm{~s}$ ( $\sim 81 \%$ of time) [10]. The trigger was provided externally by the CPU at a rate of $\sim 20 \mathrm{~Hz} .258,592$ events were recorded, each of one composed by 128 frames (Gate Time Units, GTU) of $2.5 \mu \mathrm{s}$ each, for a total of $\sim 83 \mathrm{~s}$ of acquisition time, distributed uniformly along the flight, allowing to get a detailed temporal evolution of the light intensity on the various locations.

The front-end electronics and the multi-anode photo-multipliers (MAPMT) behaved rather well. Only 1 over 9 Elementary Units (EC) had a failure. In total only 5 over 36 MAPMTs could not be used for data analysis. A detailed calibration of the 36 multi anode photo-multipliers (MAPMTs) for a total of 2,304 pixels was performed before flight and repeated after recovering the instrument. Details are reported in [11]. Among the active pixels, about 25\% of them were chosen, based on their performance, to get an accurate measurement of the UV background intensity. An detailed modelling of the electronics response is currently on-going to estimate at best the detector sensitivity and the photon-counting rate, subtracting the electronic noise of the system [12].

The optics performance (global efficiency and point spread function) was calibrated before and after flight at IRAP Toulouse. The point spread function was verified during flight by using led and flasher images. Details are reported in [13].

A bi-spectral Infrared camera operated as a stand-alone device during the flightto obtain the Cloud Top Height (CTH) and cloud coverage in the Field of View (FoV) by using two Long Wave InfraRed (LWIR) bands centred at $10.8 \mu \mathrm{m}$ and at $12 \mu \mathrm{m}$ [4]. During the flight about 350 images were recorded. The performance of the camera is under evaluation and the calibrated data will be used to calculate the $\mathrm{CTH}$ of all the clouds in the FoV of the camera [14].

\section{UV background measurement}

The main scientific objective of the EUSO-Balloon flight is the absolute measurement of the UV background intensity. This is relevant for JEM-EUSO as it is one of the key parameters to 


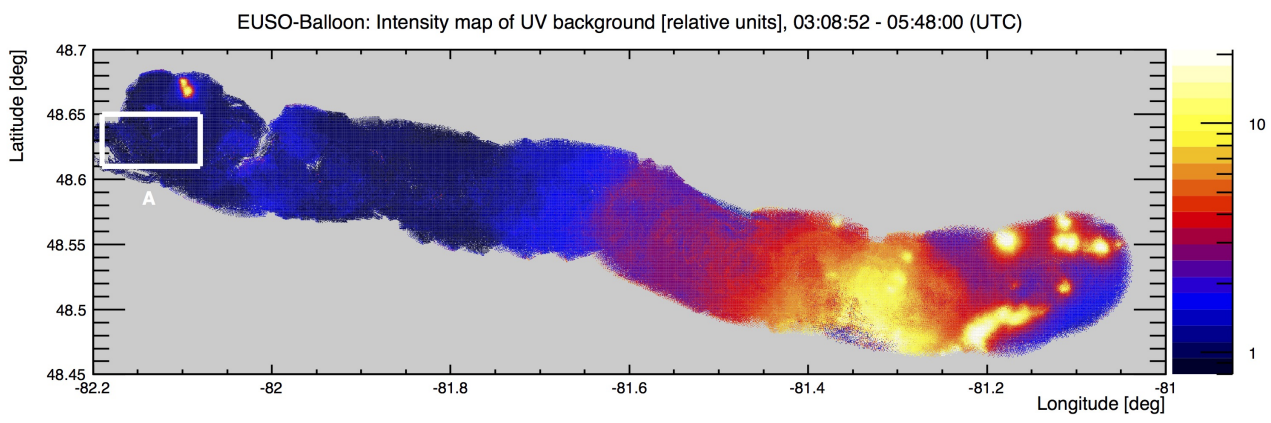

Figure 3: The intensity map of UV background in logarithmic scale (relative units). The bright areas with high intensities represent artificial light in Timmins with neighborhoods, mines, and airport. The red and light blue areas are related to cloud coverage (see Fig. 4). The dark blue areas indicate the lowest values of UV background. The displayed values are relative to the mean value of UV background intensity over reference area "A" [19].

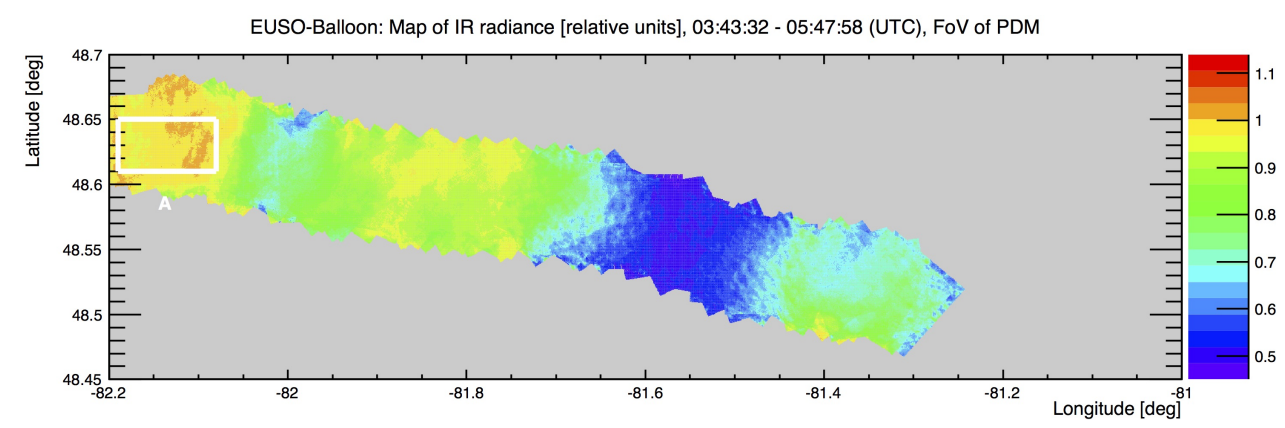

Figure 4: The map of IR radiance (relative units). The map is created by averaged values for particular positions. The values were changing in time due to movement of clouds and motion of EUSO-Balloon. The displayed values are relative to the mean value of IR radiance over reference area "A" [19].

estimate the exposure curve as a function of energy [15]. Measurements have been performed in the past by BaBy [16], NIGHTGLOW [17] and Sakaki et al. [18], however, EUSO-Balloon uses an optical refractive system with very fine spatial and temporal resolutions which allows a much better determination of the space and time variations of the UV intensity. A detailed description of the analysis and results to infer the UV background intensity of EUSO-Balloon is reported in [19]. The conversion of the digital counts of the pixels into UV intensity has to take into account many aspects, among them the most relevant are: entrance aperture of the optics and its throughput, MAPMT detection and filter efficiencies, pixel's field of view and GTU duration. Many of these parameters are wavelength dependent. An accurate determination of all these parameters is currently on going. The results obtained so far have to be considered, therefore, preliminary.

The relative intensity map of UV background in logarithmic scale is shown in Fig. 3. The bright areas with high intensities represent artificial light in the city of Timmins and its neighbourhoods, mines, and airport. The red and light blue areas are related to cloud coverage (see Fig. 4). The dark blue areas indicate the lowest values of UV background. Analysis of the clear sky region showed that there are no significant variations in the UV background intensity from different ground surfaces, such as forest and lakes. The intensities of UV emission from pixels with different 
surfaces are the same within the measurement uncertainties. In general, there is an anti-correlation between the UV flux from a given direction and the IR radiance from the same direction in presence of clouds, where the UV intensity can rise till by a factor of two, while this effect is not present in case of clear-sky conditions. Qualitative explanation for the anti-correlation is that clouds with higher optical depth are more efficient in scattering the UV radiation and producing an albedo which increases the overall intensity of the UV background in the cloudy pixels. UV radiation is absorbed in the atmosphere and higher altitude clouds have higher albedo (at equal optical depth). Higher clouds are also colder and produce lower IR radiance. In general, combination of the measurement of IR emission and UV albedo of the clouds provides a tool for characterisation of the clouds, which should improve the quality of reconstruction of EAS occurring in the cloudy sky. In presence of urban areas the UV light intensity rises even higher than 10 times compared to green areas. This relative behaviour is essentially in agreement with measurements performed by Baby.

\section{Helicopter events, IR data and other analyses}

The helicopter events revealed to be extremely useful to understand the system's performance (optics, photo-detector and front-end electronics) and to test the capability of EUSO-Balloon to detect and reconstruct EAS-like events. The EUSO-Balloon configuration has been implemented inside the JEM-EUSO OffLine package [20]. Laser tracks are used to test the reconstruction algorithms [6]. The analysis, currently in use, is based on the geometry of the triggered pixels to constrain the evolution of the event on the so called Shower Detector Plane (SDP). A first guess of the shower geometry is considered, then the difference between the expected and the observed time the signal goes through the pixels is used to further constrain the geometry. In this way, the distance of closest approach, $R_{P}$, and the angle from horizontal to $R_{P}, \Psi_{0}$, are determined to provide information on the shower axis. The geometry is better defined by using the known position of the helicopter.

The typical time fit of a laser event and the direction reconstruction are shwon in Fig. 5. Details can be found in [6]. It is important to remember that the read-out period of $2.5 \mu$ is optimised for JEM-EUSO, which is expected to detect EAS at $\sim 400 \mathrm{~km}$ distance, instead of $\sim 35 \mathrm{~km}$ as in case of EUSO-Balloon. The fact that EAS-like tracks can be reconstructed also in EUSO-Balloon is quite promising in view of JEM-EUSO.

The EUSO-Ballon data have been analysed to test the performance of the First Level Trigger of JEM-EUSO [21]. Around 300 laser tracks were detected. The system showed to be flexible enough to adapt its response to the very variable background conditions during the night. The rate of triggers on UV background, clouds, cities, etc. satisfies JEM-EUSO's requirements. No EAS events were recognised so far among the triggers. However, this is expected as the data acquisitions were based on a synchronised clock.

An analysis is on going to investigate the sensitivity of the instrument to coherent fluctuations of the UV background over a large area up to the entire field of view, on timescales ranging from a few microseconds to a few seconds and search for significant fluctuations [22].

The IR camera images during time that the flashers were operated under the balloon are being analysed. The optical depth of clouds is inferred by comparing the luminosity of the flashers with the signal recorded by EUSO-Balloon both in clear and not clear conditions. An accurate method 

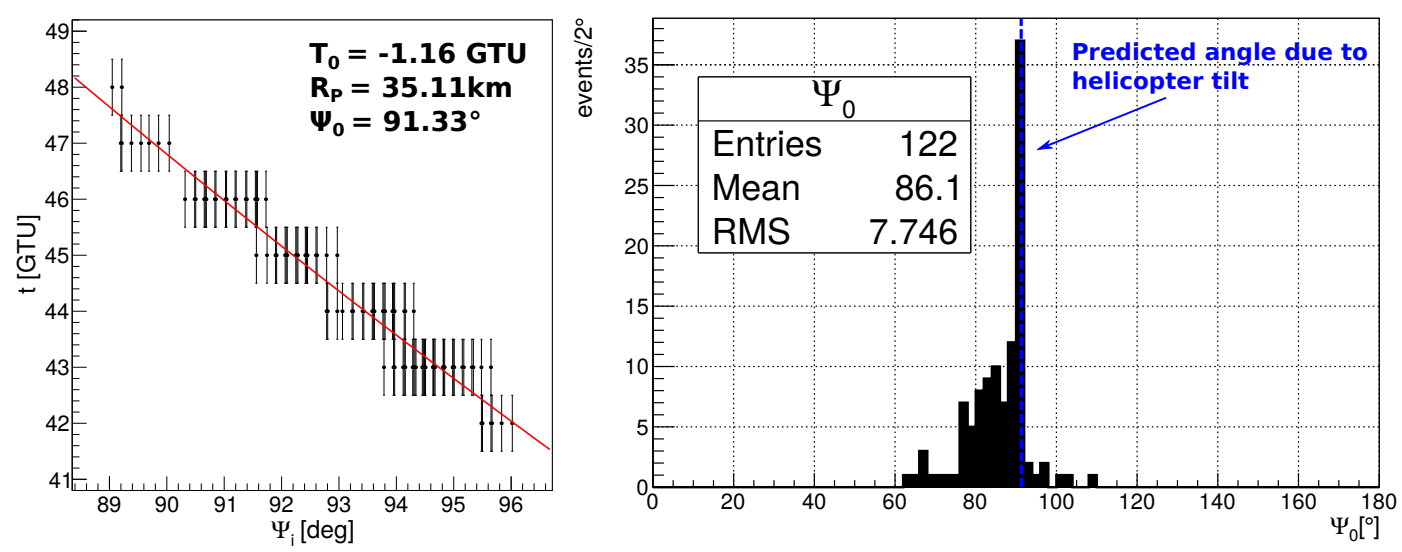

Figure 5: Left side: Time fit of laser event, with constant timing error of 0.5GTUs. Right side: Zenith angle reconstruction of the helicopter laser shots [6].

for extracting Cloud Top Heights via vertical profiles predicted by the Weather Research and Forecasting (WRF) model [23] is developed in [7]. The reliability of this model for the modelling of atmospheric properties has been tested in [8].

\section{Conclusions and Perspectives}

The EUSO-Balloon flight in 2014 was very successful. A full scale end-to-end test of most of the key technologies and instrumentation of JEM-EUSO detectors was performed. A detailed and precise measurement of the UV background in different atmospheric and ground conditions was achieved. The analysis is in progress to provide the absolute intensities. The cross-correlation of the UV and IR maps allowed detecting the phenomenon of the night-time UV albedo of clouds. This result is very useful in JEM-EUSO perspective as it will help selecting high-quality cosmic ray data. The detection of laser events proved the feasibility of the observation of EAS-like events.

The EUSO-Balloon is currently being refurbished for a second flight of much longer duration [24]. This second flight will host the First Level Trigger of JEM-EUSO whose performance revealed to satisfy the scientific requirements and proved the capability of detecting EAS-like events around a few $10^{18} \mathrm{eV}[21,25]$. The quality of their reconstruction is under evaluation [26]. Moreover, a diffractive lens and front-end electronics of higher quality are under development to improve the dynamic range of the photo-detector and the spot spread function of the optics. In the meanwhile, an endurance test of the same technologies as well as the observation of real EAS above $10^{18} \mathrm{eV}$ is on going with the EUSO-TA telescope [27].

Acknowledgment: This work was partially supported by Basic Science Interdisciplinary Research Projects of RIKEN and JSPS KAKENHI Grant (22340063, 23340081, and 24244042), by the Italian Ministry of Foreign Affairs and International Cooperation, by the 'Helmholtz Alliance for Astroparticle Physics HAP' funded by the Initiative and Networking Fund of the Helmholtz Association, Germany, and by Slovak Academy of Sciences MVTS JEMEUSO as well as VEGA grant agency project 2/0076/13. Russia is supported by the Russian Foundation for Basic Research Grant No 13-02-12175-ofi-m. The US is supported by the NASA grants NNX13AH55G, NNX13AH53G. The Spanish Consortium involved in the JEM-EUSO Space Mission is funded by MICINN \& MINECO under the Space 
Program projects: AYA2009-06037-E/AYA, AYA-ESP2010-19082, AYA-ESP2011-29489-C03, AYA-ESP2012-39115C03, AYA-ESP2013-47816-C4, MINECO/FEDER-UNAH13-4E-2741, CSD2009-00064 (Consolider MULTIDARK) and by Comunidad de Madrid (CAM) under projects S2009/ESP-1496 \& S2013/ICE-2822.

\section{References}

[1] J.H. Adams Jr. et al. (JEM-EUSO Coll.), Experimental Astronomy (2015), submitted.

[2] T. Ebisuzaki et al. (JEM-EUSO Coll.), Adv. Space Res. 53 (2014) 1499.

[3] P. von Ballmoos et al. (JEM-EUSO Coll.), Proc. 34th Int. Cosmic Ray Conf. (Den Haag), \#0725 (2015).

[4] M.D. Rodríguez Frías et al. (JEM-EUSO Coll.), Proc. 34th Int. Cosmic Ray Conf. (Den Haag), \#1309 (2015).

[5] J.H. Adams Jr. et al. (JEM-EUSO Coll.), Proc. 34th Int. Cosmic Ray Conf. (Den Haag), \#0429 (2015).

[6] J. Eser et al. (JEM-EUSO Coll.), Proc. 34th Int. Cosmic Ray Conf. (Den Haag), \#0860 (2015).

[7] A. Merino et al. (JEM-EUSO Coll.), Proc. 34th Int. Cosmic Ray Conf. (Den Haag), \#0979 (2015).

[8] I. Tabone et al. (JEM-EUSO Coll.), Proc. 34th Int. Cosmic Ray Conf. (Den Haag), \#0889 (2015).

[9] The Pierre Auger Collaboration, P.Abreu et al., Techniques for measuring aerosol attenuation using the Central Laser Facility at the Pierre Auger Observatory, JINST 8, p04009

(2013),[arXiv:13035576v].

[10] G. Osteria et al. (JEM-EUSO Coll.), Proc. 34th Int. Cosmic Ray Conf. (Den Haag), \#1025 (2015).

[11] C. Moretto et al. (JEM-EUSO Coll.), Proc. 34th Int. Cosmic Ray Conf. (Den Haag), \#1016 (2015).

[12] S. Dagoret Campagne et al. (JEM-EUSO Coll.), Proc. 34th Int. Cosmic Ray Conf. (Den Haag), \#0625 (2015).

[13] C. Catalano et al. (JEM-EUSO Coll.), Proc. 34th Int. Cosmic Ray Conf. (Den Haag), \#0717 (2015).

[14] J. Fernández Soriano et al. (JEM-EUSO Coll.), Proc. 34th Int. Cosmic Ray Conf. (Den Haag), \#1008 (2015).

[15] J.H. Adams Jr. et al. - JEM-EUSO Collaboration, Astroparticle Physics 44 (2013) 76.

[16] S. Giarruso et al., Proc. 28th Int. Cosmic Ray Conf. (Tsukuba), 2 (2003) 849.

[17] L.M. Barbier et al., Astroparticle Physics 22 (2005) 439.

[18] N. Sakaki et al., Proc. 30th Int. Cosmic Ray Conf. (Merida), 5 (2007) 965.

[19] S. Mackovjak et al. (JEM-EUSO Coll.), Proc. 34th Int. Cosmic Ray Conf. (Den Haag), \#1302 (2015).

[20] B. Panico et al. (JEM-EUSO Coll.), Proc. 34th Int. Cosmic Ray Conf. (Den Haag), \#1155 (2015).

[21] G. Suino et al. (JEM-EUSO Coll.), Proc. 34th Int. Cosmic Ray Conf. (Den Haag), \#0925 (2015).

[22] A. Jung et al. (JEM-EUSO Coll.), Proc. 34th Int. Cosmic Ray Conf. (Den Haag), \#0971 (2015).

[23] W.C. Skamarock et al., NCAR technical note (2008).

[24] L. Wiencke et al. (JEM-EUSO Coll.), Proc. 34th Int. Cosmic Ray Conf. (Den Haag), \#0816 (2015).

[25] S. Bacholle et al. (JEM-EUSO Coll.), Proc. 34th Int. Cosmic Ray Conf. (Den Haag), \#0632 (2015).

[26] F. Fenu et al. (JEM-EUSO Coll.), Proc. 34th Int. Cosmic Ray Conf. (Den Haag), \#0639 (2015).

[27] M. Casolino et al. (JEM-EUSO Coll.), Proc. 34th Int. Cosmic Ray Conf. (Den Haag), \#0854 (2015). 